Conclusion It is feasible to introduce standards to increase the availability of healthier snack products in vending machines and assess changes in purchased snacks without substantially affecting sales.

\section{OP42 ASSOCIATIONS BETWEEN GLOBALISATION AND THE DOUBLE BURDEN OF MALNUTRITION: A MULTI-LEVEL ANALYSIS OF REPEATED CROSS-SECTIONAL DATA FROM 55 LOW- AND MIDDLE-INCOME COUNTRIES 1992-2018}

Paraskevi Seferidi*, Thomas Hone, Christopher Millett. Public Health Policy Evaluation Unit, School of Public Health, Imperial College London, London, UK

\subsection{6/jech-2021-SSMabstracts.42}

Background Globalisation can transform food systems and contribute to a nutrition transition from local diets to westernised diets high in ultra-processed foods. This is likely to influence malnutrition in all its forms, however there is no evidence on its association with the double burden of malnutrition (DBM), i.e. the co-existence of overnutrition and undernutrition within the same individual, household, or population. This analysis investigates the association between DBM, expressed as a stunted child and overweight mother in the same household, and economic, social, and political globalisation, and how these differ by country income and household wealth.

Methods We pooled anthropometric and demographic data for 1,131,069 children aged under 5 years and their mothers, aged 15-49 years, from 189 Demographic and Healthy Surveys (DHS) conducted between 1992 and 2018 in 55 low- and middle-income countries (LMICs). These were combined with country-level data on globalisation, using the KOF Globalisation Index de jure, and gross national income (GNI) from the World Bank. Multi-variate associations between the DBM and globalisation were tested using logistic regression with country and time fixed-effects and robust standard errors clustered by country. Interactions between globalisation, household wealth index and GNI were also included in the model.

Results The DBM was distributed unequally across groups of affluence in our sample, with higher probability of DBM among rich households of LMICs at low GNI levels, and among poor households of LMICs at high GNI levels. We identified that economic globalisation was associated with higher odds of children being stunted and having an overweight mother among the poorest but not the richest wealth quintiles (OR; 95\% CI: 1.52; 1.18-1.97 for poorest quintile). These associations were attenuated as country income increased. Social globalisation was also associated with higher odds of children being stunted and having an overweight mother, although this did not vary significantly by household wealth or country income (OR; 95\% CI: 1.43; 1.18-1.74). No associations were identified between political globalisation and the DBM.

Conclusion This analysis suggests that the DBM is affecting rich households of poorer LMICs and poor households of richer LMICs the most. It also indicates that economic and social globalisation are associated with higher DBM, although the impacts of economic globalisation are mostly realised by the world's poorest.
Friday 17 September

Womens Health, $09.00-11.30$

\section{OP43 MEDIATORS OF SOCIOECONOMIC DIFFERENCES IN PRETERM BIRTH: A SYSTEMATIC REVIEW OF THE EVIDENCE}

${ }^{1}$ Philip McHale*, 'Gillian Maudsley, ${ }^{1}$ Andy Pennington, 'Daniela Schlüter, ${ }^{1}$ Ben Barr, ${ }^{2}$ Shantini Paranjothy, 'David Taylor-Robinson. 'Department of Public Health, Policy and Systems, University of Liverpool, Liverpool, UK; ${ }^{2}$ Aberdeen Health Data Science Research Centre, University of Aberdeen, Aberdeen, UK

\subsection{6/jech-2021-SSMabstracts.43}

Background Approximately $8 \%$ of births in the UK are preterm, and rates have been increasing for the past decade. There are significant inequalities in the prevalence of preterm birth, with some estimates showing 50\% higher prevalence in mothers with low socioeconomic status (SES). Understanding and explaining how low SES increases the risk of preterm birth, will be important to reduce these inequalities.

Methods Systematic review of quantitative observational studies of mediation of the relationship between maternal SES and preterm birth. Searches were conducted from five databases covering January 2000 to June 2020, and all were identified for dual screening based on title and abstract review. Inclusion criteria was comparison across SES, preterm birth or gestational age as outcome, and study of mediation of non-genetic variables. All included studies were citation searched. All studies were narratively synthesised and quality assessed.

Results Initial searches identified 58 studies for full-text screening, of which 13 met inclusion criteria, and citation searching identified four more studies. A final sample of 17 studies from 11 countries (five from North America, 11 from Europe, and one each from Africa, the Middle East and South America; one study covered three European countries) were identified for analysis, covering study periods between 1980 and 2013. The majority (10/17) used the difference-of-coefficients approach and estimated proportion mediated. Other methods included multiplication of coefficients and counterfactual approaches. Forty-eight mediators (or groups of mediators) were examined. SES was measured most frequently by maternal education (7/17). All studies used a categorisation of preterm birth. The most frequently examined mediator was smoking $(8 / 17)$, with the proportion estimated ranging from $2 \%$ to $43 \%$. A residual direct effect of SES on preterm birth not via mediating exposures studied remained in over half of these studies. Four studies examined mediation via maternal health (range 6\% to 39\%), BMI (range $2 \%$ to $17 \%$ ), and depression and anxiety (range from no effect to $44 \%$ ).

Discussion Our review suggests that inequalities in preterm birth are in part explained by unequal exposure to risk factors across socioeconomic strata. However, limitations in the methods to assess mediation, reporting of mediation results, and measures of SES used mean it is difficult to meaningfully combine findings. A major limitation is the lack of consideration for the theoretical basis for mediation in the data, such as the underlying assumptions. Despite these limitations, these findings highlight potential intervention points for reducing preterm birth inequalities. 